\title{
Resection of a large left opercular cavernoma in a child using neuronavigation
}

\author{
Ricardo Santos de Oliveira ${ }^{1}$, Vinícius Marques Carneiro ${ }^{1}$, Kaliny Batista de Oliveira ${ }^{2}$, Érica Sany Brito \\ Oliveira Costa, Matheus Fernando Manzolli Ballestero
}

\begin{abstract}
1 Division of Neurosurgery, CIN / Hospital São Lucas, Ribeirão Preto, Brazil

2 President Antônio Carlos Faculty ITPAC Porto Nacional (ITPAC Porto), Porto Nacional - TO, Brazil

${ }^{3}$ State University of Southwestern Bahia (Uesb), Candeias - BA, Brazil 4 Department of Medicine, Federal
University of São Carlos, São Carlos,
Brazil
\end{abstract}

To whom correspondence should be addressed: Ricardo Santos de Oliveira, MD, PhD

e-mail:

rsoliveira30@gmail.com

Available at:

http://www.archpedneurosurg.com.br/
The insular lobe is a complex structure constituting anatomic, cytoarchitectonic, and functional interface between the allocortex and the neocortex. This area is a part of a larger system that includes the orbitofrontal, temporopolar, and insular regions, constituting the paralimbic system or mesocortex. For a long time, the issue in studying this entity accounted for the poor understanding of its precise functionality. Because of the technical complexity in approaching and dissecting this region, very few neurosurgeons attempted surgery of the insula. Particularly on the dominant side, the natural history of these lesions must be carefully balanced with the surgical morbidity involved in their resection. Surgical treatment of cavernomas arising around the insula (especially in dominant cerebral hemisphere) is challenging in reason of the proximity to the internal capsule and lenticulostriate arteries. The advent of image guidance systems and intraoperative mapping of the subcortical language pathways has broadened the surgical indications for these lesions. In the case reported here we removed a cavernous angioma of the left dominant operculum in a child with the aid of a neuronavigation system guidance.

Keyworks: Cavernoma, neuronavigation, insula, left operculum

\section{CLINICAL CASE}

This 13-year-old female with a generalized seizure in April 2019. She was admitted to our institute after left peri-insular cavernous malformation (CM) was diagnosed based on results of MR imaging (Figure 1 ).

The lesion measured approximately $20 \mathrm{~mm}$, was located at a depth of $21 \mathrm{~mm}$, and was surrounded by a typical hemosiderin-stained rim on T2-weighted images. On admission, the patient was neurologically intact. We decided to remove the lesion by using an image-guided procedure.

\section{OPERATION}

The surgical approach was tailored using a Brain Lab? neuronavigation system (Figure 2). The Sylvian fissure was opened partially under the operating microscope, following an approach defined on the pre-operative plans.

The cortex overlying the lesion was identified by recognizing its morphological pattern. The cavernoma was quickly reached by splitting the fissure. The cavernoma was totally removed. Pathological examination confirmed the diagnosis of cavernous hemangioma. No neurological deficits or seizures were observed after surgery, and the postoperative course was uneventful in all patients (Figure 3 ). 


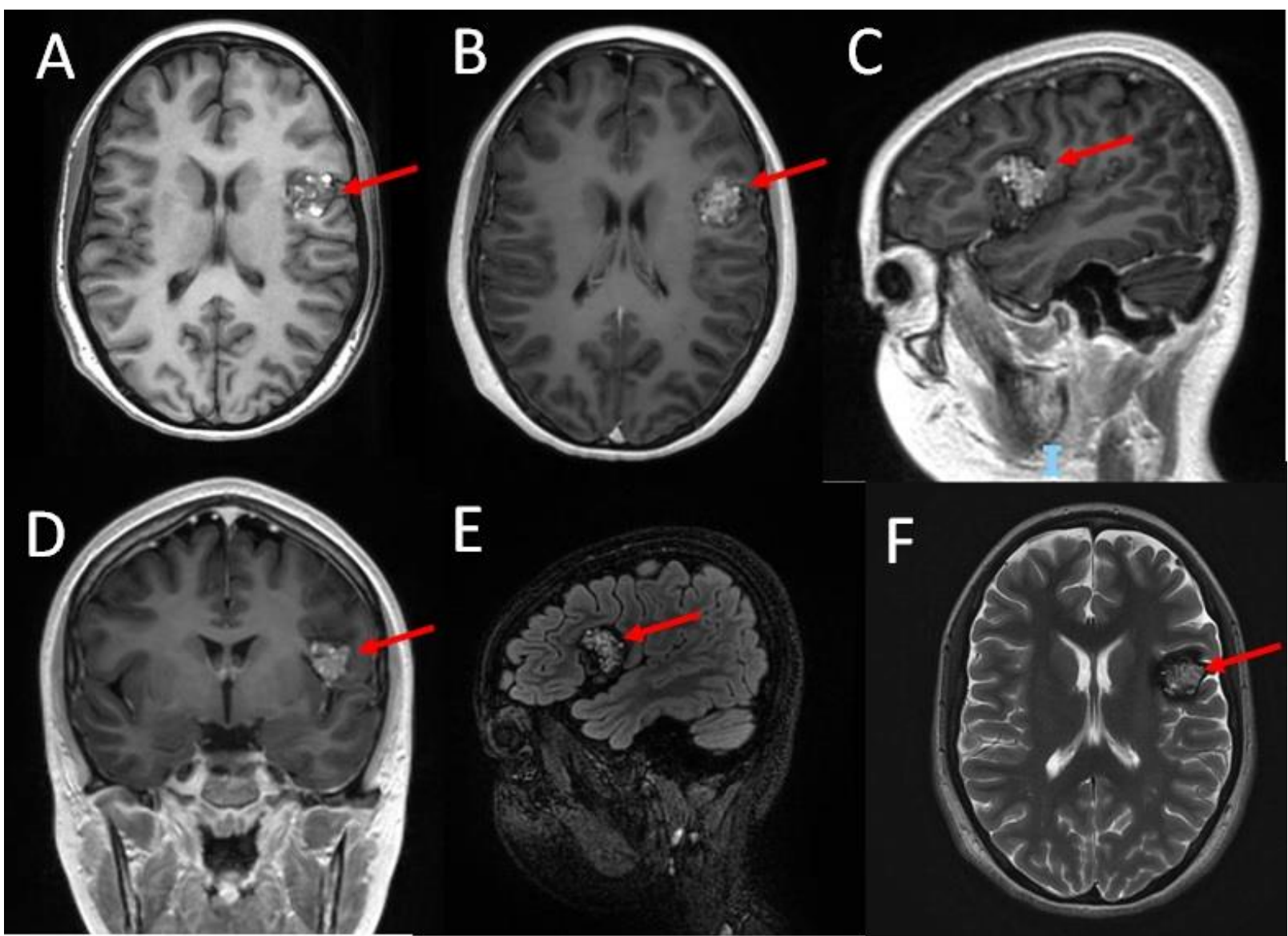

Figure 1 - Pre operative MRI showing lesion (red arrow) aspect in T1-weighted sequences, axial non contrasted (A), axial contrasted (B), sagittal contrasted (C) and coronal contrasted (D). Flair Sagittal (E). T2 axial (F).

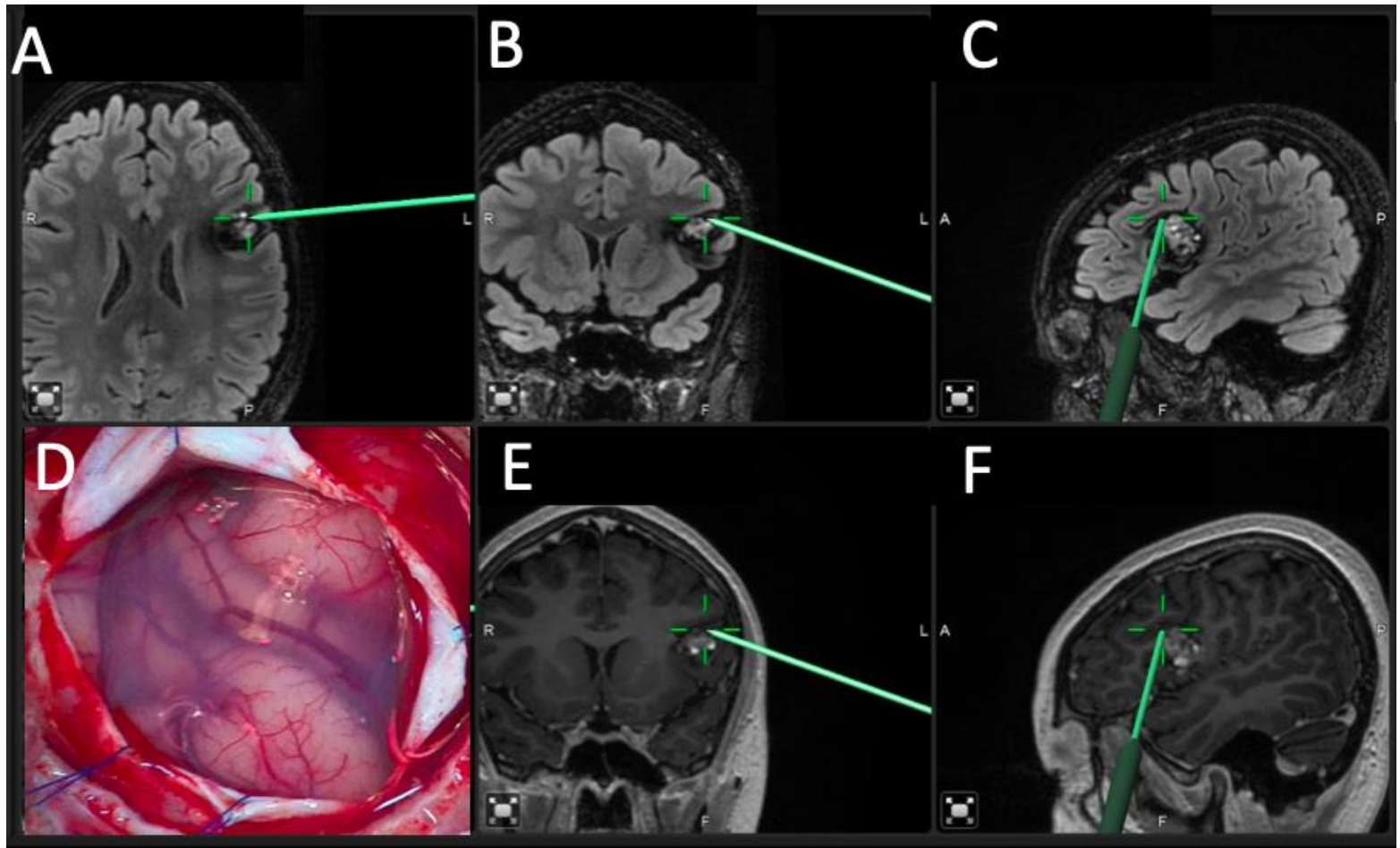

Figure 2 - Intraoperative use of Brain Lab] neuronavigation system to perform a small craniotomy (A, B, C, E and F). Cortical aspect of guided craniotomy (D). 


\section{Resection of a large left opercular cavernoma in a child using neuronavigation}

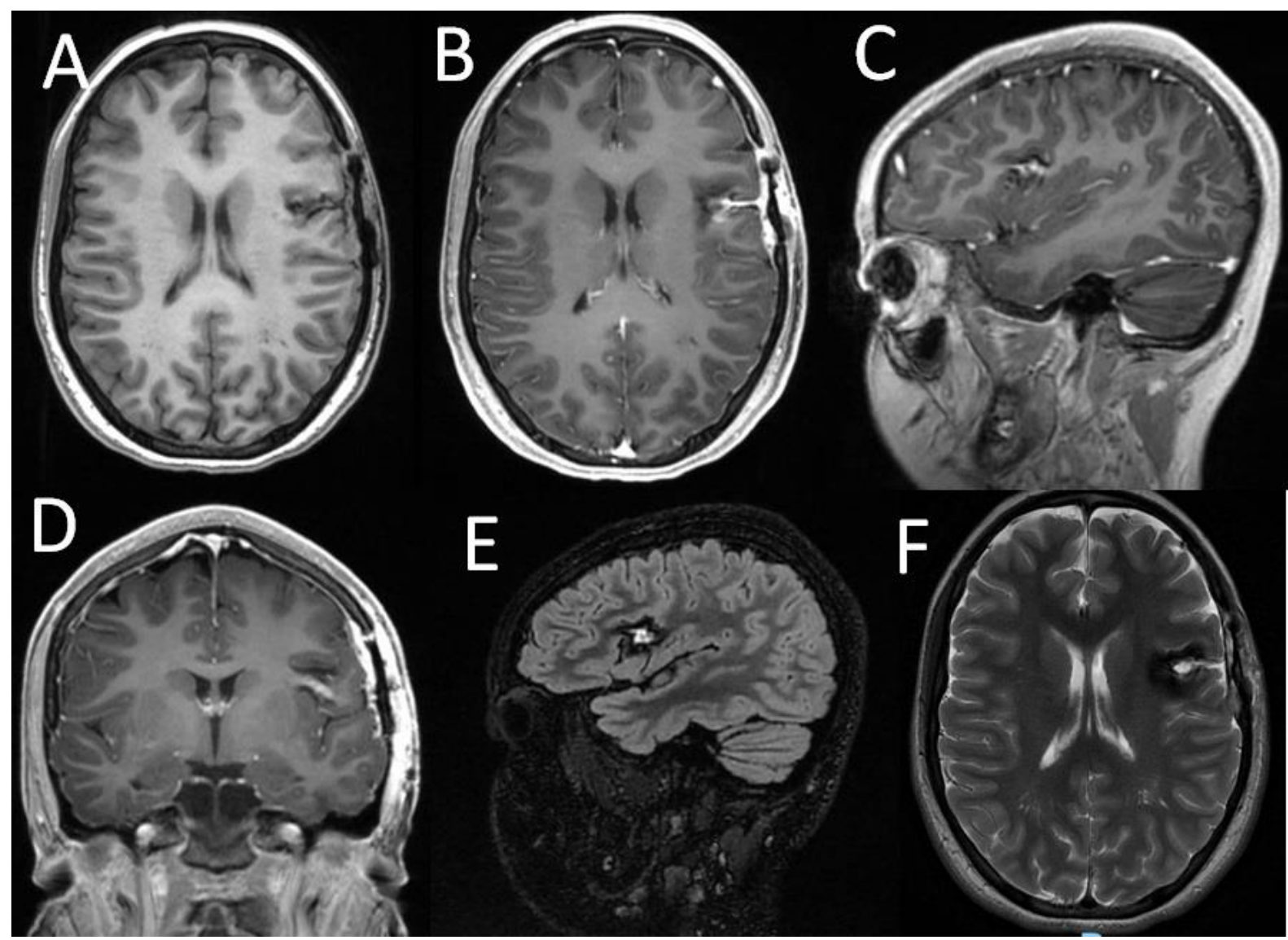

Figure 3 - Post operative MRI showing lesion aspect in T1-weighted sequences, axial non contrasted (A), axial contrasted (B), sagittal contrasted (C) and coronal contrasted (D). Flair Sagittal (E). T2 axial (F).

\section{DISCUSSION}

$\mathrm{CM}$ is one of the congenital vascular malformations affecting the central nervous system (CNS) that may remain asymptomatic for a long time or may produce clinical manifestations. Presence or absence of hemorrhage is important to decide how to manage the patients. The risk of bleeding is higher after the first event, $22.9 \%$ against 0.25 $3.8 \%$ without prior hemorrhage. However, without a documented episode of hemorrhage, treatment should be based on the precise location of the cavernoma, patient's age, symptoms, and outcome expectation $(4,5)$.

Surgical treatment should be indicated to the majority of patients that suffered hemorrhage and to those which surgical risk was considered low.

Lesionectomy alone showed favorable results (6). In a series of patients submitted to lesionectomy of supratentorial CMs, a strict relationship was found between the duration of seizure history before the surgical treatment and the cure of epilepsy with the surgery. Cohen et al. (6) showed that all patients that presented one preoperative seizure or less than two months of history did not have any seizures after surgery; 75 to $80 \%$ of patients with two to five preoperative seizures or 2 to 12 months of history also had not any seizure after surgery. In addition, $50 \%$ of patients with more than five seizures or more than 12 months of history were postoperatively seizure free. Although resection of the hemosiderin stained tissue that surrounds the lesion was not proved to be of better effectiveness, it was adopted by several studies when $\mathrm{CMs}$ are placed in noncritical areas, including the present one.

The management of $\mathrm{CM}$ of the brain is markedly influenced by the location of the lesions themselves. In the last decade, resection of cavernomas arising in the dominant insular lobe has been deemed safe only with the guidance of neuronavigation $(2,3)$

Image guidance during surgery for peri-insular cavernomas provides high accuracy for lesion targeting and permits excellent anatomic orientation. Accordingly, safe exposure can be obtained because of a tailored dissection of the sylvian fissure and minimal insular corticotomy (7).

In conclusion, although surgical treatment of symptomatic $\mathrm{CM}$ still remains controversial when the lesion 


\section{Resection of a large left opercular cavernoma in a child using neuronavigation}

is located in critical brain structures, the risk of morbidity due to the natural history of this malformation is nevertheless higher in children. Thus, we advocate careful consideration of the possibility of an extensive surgical resection using combined modern technologies, particularly in young patients.

\section{COMPLIANCE WITH ETHICAL STANDARDS}

Disclosures: The authors declare that they have no conflict of interest. All authors certify that they have no affiliations with or involvement in any organization or entity with any financial interest or non-financial interest in the subject matter or material discussed in this manuscript.

Ethical approval: The patient's guardian has consented to the submission of the case report and photographs to the journal. Detailed informed consent was provided.

Funding: The cost relating to professional proofreading was funded by the researcher. They were no other costs. No funding was received for this research.

\section{REFERENCES}

1. Leal PR, Houtteville JP, Etard O, Emery E. Surgical strategy for insular cavernomas. Acta Neurochir (Wien). 2010 Oct;152(10):1653-9. doi: 10.1007/s00701-010-0710-x. Epub 2010 Jun 19. PMID: 20563609.
2. Tirakotai W, Sure U, Benes L, Krischek B, Bien S, Bertalanffy $H$. Image-guided transsylvian, transinsular approach for insular cavernous angiomas. Neurosurgery. 2007 Jul;61(1 Suppl):42330; discussion 430-1. doi: 10.1227/01.neu.0000279234.81320.eb. PMID: 18813148.

3. Pasqualin A, Meneghelli P, Giammarusti A, Turazzi S. Results of surgery for cavernomas in critical supratentorial areas. Acta Neurochir Suppl. 2014;119:117-23. doi: 10.1007/978-3-319-024110_20. PMID: 24728644.

4. Detwiler PW, Porter RW, Zabramski JM, Spetzler RF. De novo formation of a central nervous system cavernous malformation: implications for predicting risk of hemorrhage. Case report and review of the literature. J Neurosurg 1997;87:629632.

5. Amato MC, Madureira JF, Oliveira RS. Intracranial cavernous malformation in children: a singlecentered experience with 30 consecutive cases. Arq Neuropsiquiatr. 2013 Apr;71(4):220-8. doi: 10.1590/0004-282x20130006. PMID: 23588283.

6. Cohen DS, Zubay GP, Goodman RR. Seizure outcome after lesionectomy for cavernous malformations. J Neurosurg 1995;83:237-242

7. Duffau H, Fontaine D. Successful resection of a left insular cavernous angioma using neuronavigation and intraoperative language mapping. Acta Neurochir (Wien). 2005 Feb;147(2):205-8; discussion 208. doi: 10.1007/s00701-004-0357-6. Epub 2004 Aug 30. PMID: 15338338. 